\title{
Research Paper: Estimating Chronological Age by Dental Measurements on the Periapical Radiographs in Iranian Adults
}

\author{
Mitra Akhlaghi $^{1,2}$ (D), Zahra Ghoncheh ${ }^{3 *}$ (D) Lida Hatami² (D)
}

1. Department of Forensic Medicine, School of Medicine, Tehran University of Medical Sciences, Tehran, Iran

2. Legal Medicine Research Center, Legal Medicine Organization, Tehran, Iran.

3. Department of Oral and Maxillofacial Radiology, School of Dentistry, Tehran University of Medical Sciences, International Campus, Tehran, Iran.

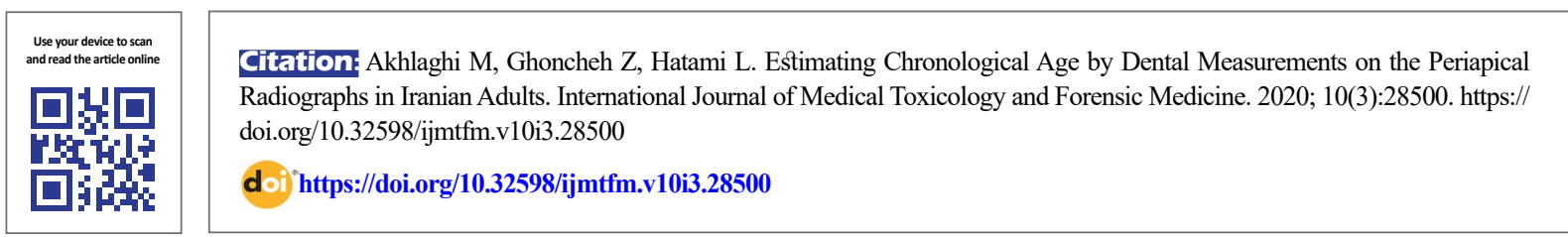

\section{(1) (9)}

Article info:

Received: 04 Jan 2020

First Revision: 24 Jan 2020

Accepted: $01 \mathrm{Jul} 2020$

Published: 13 Oct 2020

\section{Keywords:}

Radiography, Forensic dentistry, Anthropology

\section{A B S T RACT}

Background: This study aimed to assess the accuracy of chronological age estimation based on dental measurements made on Periapical (PA) radiographs of an Iranian adult population.

Methods: This study evaluated 90 parallel PA radiographs of sound maxillary canine teeth of 39 males and 51 females. Tooth length, root length, pulp length, pulp width and root width at points A, B and C according to the Kvaal's method were measured on PA radiographs using Scanora software. Data were analyzed using SPSS.

Results: Maximum Root width at point A had the highest accuracy for sex estimation (77.7\%). A significant correlation was noted between maximum pulp width at points $\mathrm{B}$ and $\mathrm{C}$ with age and a regression formula for age estimation was obtained.

Conclusion: Maximum pulp width at points B and C can be used for age estimation in adult population beside other parameters.

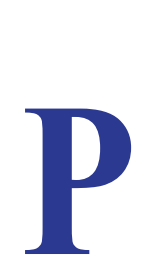

\section{Introduction}

ersonal identification is a fundamental task in forensic medicine. Age estimation could greatly help in personal identification. However, age estimation becomes more difficult by aging [1]. In infants and babies, age could be estimated based on height and weight using related reference tables and formulas.
However, the risk of errors exists in cases with malnutrition, specific diseases, or developmental anomalies [2]. Several parameters may be used for age estimation, as the remnants of bones and teeth. Teeth are resistant to postmortem changes, such as thermal alterations, microbial activity, and mechanical loads; they are the last tissue to degrade after death [3]. Thus, teeth are more reliable than bones for age estimation and are often used for this purpose.

\section{* Corresponding Author: \\ Zahra Ghoncheh, PhD.}

Address: Department of Oral and Maxillofacial Radiology, School of Dentistry, Tehran University of Medical Sciences, International Campus, Tehran, Iran.

Tel: +98 (912) 3056657

E-mail:m_ghoncheh@hotmail.com 
Physiological changes, as well as growth and development, affect the teeth in living individuals; these changes could be observed during the aging process. In Western Australia with 2.2 million population, 100 cases are referred to forensic medicine specialists annually; of them, $82 \%$ require personal identification $[1,4]$. The dental information of each individual is unique; therefore, such data could be used for personal identification [4]. Evidence suggests that dental age is closer to chronological age, compared with skeletal age; thus, dental age is more reliable for age estimation [5].

Radiography could greatly help in age estimation based on teeth. Periapical (PA) and panoramic radiography could be used for this purpose $[6,7]$. Several dental parameters, such as tooth length, root length, and pulp length, as well as dental ratios, such as maximum pulp length/root length, pulp length/tooth length, and maximum pulp width/root width according to the Kvaal's method are often measured for age estimation [8]. However, such measurements and their correlations with chronological age may vary in different populations. Thus, studies on different populations are required to calculate the most suitable and unique regression formulas for that specific population $[1,2,5]$. Thus, this study aimed to assess the accuracy of chronological age estimation based on dental measurements made on the PA radiographs of an Iranian population.

\section{Materials and Methods}

This cross-sectional analytical study evaluated the PA radiographs of patients presenting to an oral and maxillofacial radiology clinic in Tehran City, Iran, in 2017-2018.

The inclusion criteria of the study were being Iranian adults, the age of 20 years, requiring PA radiographs for dental treatments, and maxillary canine teeth (right/left) be presented on radiographs. The study exclusion criteria were poor-quality PA radiographs, dental caries, dental developmental anomalies, images with technical errors and pulp, and PA pathologies. The study participants were selected using a convenience sampling method. The sample size was calculated to be 80 assuming alpha $=0.05$ and beta $=0.05$. Considering a $10 \%$ possibility of dropouts, 90 patients were enrolled in the present research.

All PA radiographs $(\mathrm{N}=250)$ of eligible adult patients presenting to an oral and maxillofacial radiology clinic during 2017-2018 were evaluated; 51 and 39 belonged to females and males, respectively. All radiographs were captured by the same digital dental X-ray unit (MINRAY® device; Soredex, Nahkelantie 160, Finland). The following variables were measured on PA radiographs, irrespective of the patients' age and gender:

The maximum length of the tooth, root, and pulp, as well as the maximum width of pulp and root at 3 points: Cementoenamel junction (point A), mid-root (point B), and mid-point between points A and B (point C) (Figure 1). All measurements were conducted by a forensic medicine specialist under the supervision of an oral and maxillofacial radiologist using Scanora software. The intra-observer agreement was calculated to be 1 .

The data were analyzed using SPSS. Receiver Operating Characteristic (ROC) curve was drawn. Then, the Area Under the ROC Curve (AUC) was calculated to determine the gender-wise differences. Next, the sensitivity, specificity, Positive Predictive Value (PPV), and Negative Predictive Value (NPV) were calculated at the relevant cut-off points.

\section{Results}

The minimum, maximum, and mode values of the evaluated patients' age were 20,49, and 49 years, respectively. The Mean \pm SD age of the explored patients was $36.06 \pm 8.36$ years. The Mean \pm SD age was $37.13 \pm 7.5$ years in males and $35.24 \pm 8.8$ years in females. The mean age was not significantly different between males and females $(\mathrm{P}=0.29)$.

Table 1 presents the mean scores of tooth length, pulp length, root length, pulp width, and root width at points $\mathrm{A}, \mathrm{B}$, and $\mathrm{C}$ in millimeters.

The mean maximum length of the tooth, root, and pulp, as well as the mean maximum width of root and pulp at points $\mathrm{A}, \mathrm{B}$, and $\mathrm{C}$ was significant between males and females $(\mathrm{P}<0.0001, \mathrm{P}<0.002, \mathrm{P}<0.0001, \mathrm{P}<0.0001$, $\mathrm{P}<0.0001, \mathrm{P}<0.0001, \mathrm{P}<0.001, \mathrm{P}<0.0001, \& \mathrm{P}<0.002$, respectively). Thus, ROC curve was drawn to assess the prediction power of this variable for gender determination; the AUC for the mean maximum length of tooth, root, and pulp, as well as the mean maximum width of root and pulp at points $\mathrm{A}, \mathrm{B}$, and $\mathrm{C}$, was calculated to be 0.751, 0.691, 0.724, 0.828, 0.732, 0.812, 0.723, 0.836, and 0.724 , respectively.

Table 2 lists the sensitivity, specificity, PPV, and NPV of the parameters evaluated in this study for gender determination. Accordingly, the maximum accuracy belonged to maximum root width at point A (77.7\%), followed by maximum root width at point $\mathrm{C}(75.5 \%)$, root width at point $\mathrm{B}(72.2 \%)$, pulp width at points $\mathrm{A}$ and 

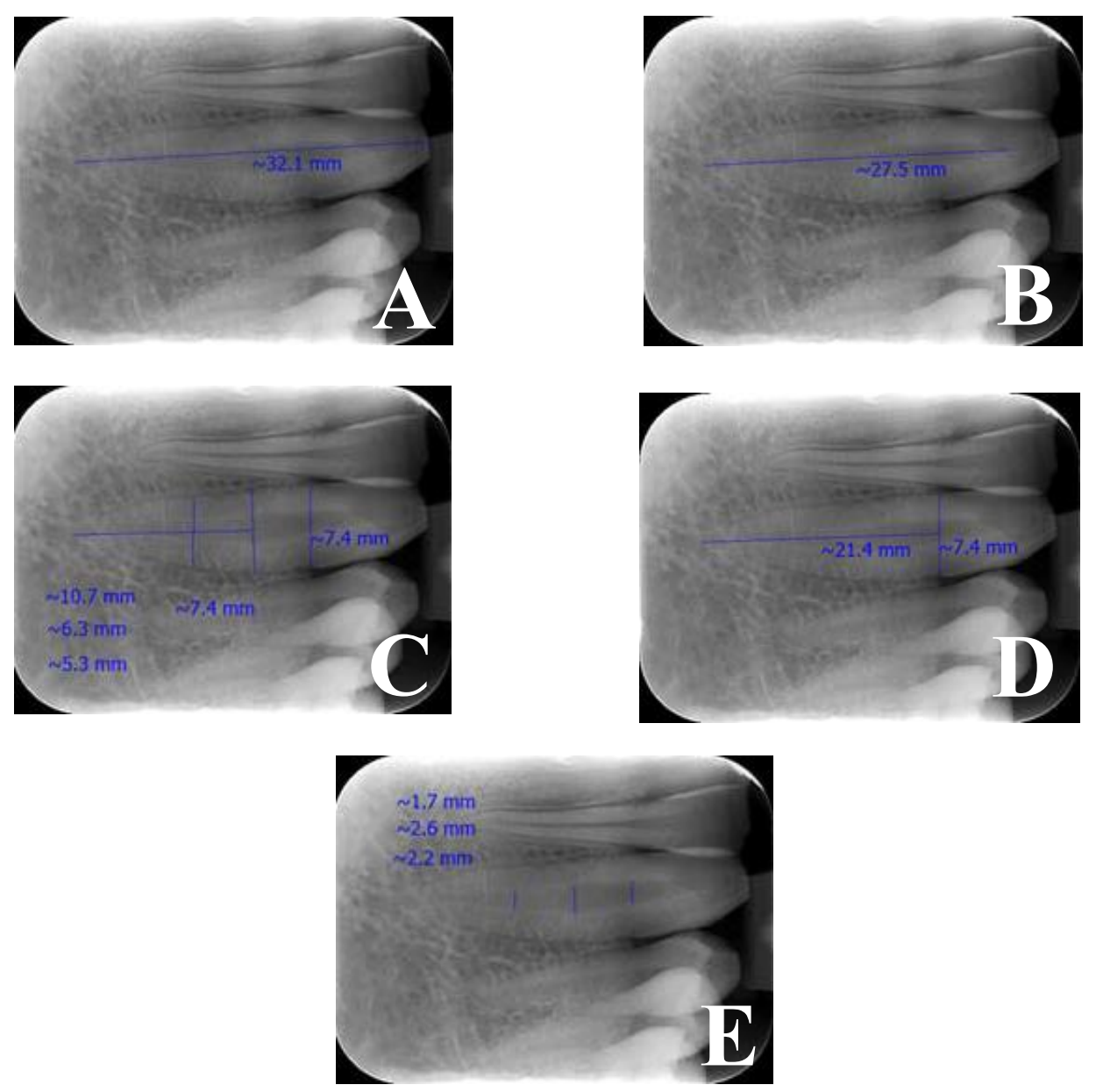

International Journal of

Medical Toxicology \& forensic Medicine

Figure 1. A. Tooth length; B. Pulp length; C. Root length; D. Root width at points A, B, and C; E. Pulp width at points A, B, and C

C (71.1\%), tooth length $(68.9 \%)$, root length and pulp length $(67.7 \%)$, and pulp width at point B $(66.6 \%)$.

No significant association was noted between gender and pulp/root length, and pulp/tooth length, pulp/root width at points $\mathrm{A}, \mathrm{B}$, and $\mathrm{C}(\mathrm{P}>0.05)$.

In the present study, significant correlations were noted between pulp width at points $\mathrm{B}$ and $\mathrm{C}$, and chronological age $(\mathrm{P}=0.003 \& \mathrm{P}=0.017$, respectively); by an increase in age, pulp width at the respective points, decreased. However, this was a poor correlation $(\mathrm{r}=0.2 \&$ $\mathrm{r}=0.3$, respectively).

The regression formula was generated for chronological age estimation based on pulp width at points $\mathrm{B}$ and $\mathrm{C}$.

\section{Pulp width at point $B$ :}

Age $(\mathrm{y})=$ Pulp width at point $\mathrm{B} \times(-8.988)+46.512$

$$
\mathrm{r}=0.308 ; \operatorname{SEE}(\mathrm{y})=8.00 ; \mathrm{P}=0.003
$$

\section{Pulp width at point $C$ :}

Age $(\mathrm{y})=$ Pulp width at point $\mathrm{C} \times(-10.711)+44.529$

$\mathrm{r}=0.251 ; \operatorname{SEE}(\mathrm{y})=8.139 ; \mathrm{P}=0.017$

\section{Discussion}

This study investigated the accuracy of chronological age estimation based on dental measurements concerning the PA radiographs of an Iranian population. Tooth length, root length, pulp length, root width, and pulp width were measured at three points; some ratios were also calculated. The obtained results indicated a significant gender-wise difference in all measurements. Root width at point A with $77.7 \%$ accuracy, had the highest accuracy for gender determination; however, pulp width at point $\mathrm{B}$ had the lowest accuracy in this area. 
Table 1. Mean tooth length, pulp length, root length, pulp width, and root width at points A, B, and C in millimeters

\begin{tabular}{|c|c|c|c|c|c|c|c|c|c|c|}
\hline \multirow{2}{*}{$\begin{array}{c}\text { Gender } \\
\text { parameter }\end{array}$} & \multicolumn{3}{|c|}{ Male } & \multicolumn{3}{|c|}{ Female } & & \multicolumn{3}{|c|}{ Total } \\
\hline & Mean $\pm S D$ & Min. & Max. & Mean $\pm S D$ & Min. & Max. & & Mean \pm SD & Min. & Max. \\
\hline Tooth length & $29.446 \pm 2.857$ & 23.4 & 35.6 & $26.906 \pm 2.347$ & 22.9 & 31.8 & $<0.0001$ & $28.007 \pm 2.860$ & 22.9 & 49 \\
\hline Root length & $19.677 \pm 2.609$ & 14.2 & 24.8 & $18.112 \pm 2.030$ & 14.5 & 22 & $<0.002$ & $18.790 \pm 2.414$ & 14.2 & 24.8 \\
\hline Pulp length & $23.362 \pm 2.563$ & 17.4 & 28.4 & $21.345 \pm 2.230$ & 16.3 & 26.1 & $<0.0001$ & $22.219 \pm 2.570$ & 16.3 & 28.4 \\
\hline Root width A & $7.003 \pm 0.559$ & 5.5 & 8.1 & $6.257 \pm 0.6004$ & 4.8 & 8.3 & $<0.0001$ & $6.580 \pm 0.688$ & 4.8 & 8.3 \\
\hline Pulp width A & $1.497 \pm 0.3609$ & 0.8 & 2.5 & $1.245 \pm 0.2866$ & 0.8 & 2.4 & $<0.0001$ & $1.354 \pm 0.3429$ & 0.8 & 2.5 \\
\hline Root width B & $6.182 \pm 0.6232$ & 4.5 & 7.6 & $5.469 \pm 0.6160$ & 4.3 & 7.8 & $<0.0001$ & $5.778 \pm 0.7109$ & 4.3 & 7.8 \\
\hline Pulp width B & $1.274 \pm 0.3338$ & 0.5 & 2.5 & $1.078 \pm 0.2101$ & 0.7 & 1.7 & 0.001 & $1.163 \pm 0.2862$ & 0.5 & 2.5 \\
\hline Root width C & $5.197 \pm 0.6124$ & 3.5 & 6.5 & $4.449 \pm 0.5065$ & 3.2 & 5.8 & $<0.0001$ & $4.773 \pm 0.6658$ & 3.2 & 6.5 \\
\hline Pulp width C & $0.864 \pm 0.2045$ & 0.4 & 1.5 & $0.735 \pm 0.1707$ & 0.4 & 1.2 & 0.002 & $0.791 \pm 0.1958$ & 0.4 & 1.5 \\
\hline
\end{tabular}

Paewinsky et al. [9] evaluated 6 maxillary and mandibular teeth on the orthopantomographs of 14- to 81-yearolds. For this purpose, Kvaal's method was employed and no significant association was detected between dental measurements and gender. Cameriere et al. [4] and Jeevan et al. [10] found no gender-wise significant difference in the pulp/tooth ratio of canine teeth on the PA radiographs of 20- to 79-year-olds.

The accuracy of root width measurement for gender determination was $77.7 \%$ at point $\mathrm{A}$; therefore, this variable may be helpful in gender determination. Pulp width at point $\mathrm{B}$ had the lowest value for gender determination. Other variables, including pulp length and root length, presented no high value for gender determination ( $<70 \%$ accuracy).

Concerning the correlation between dental variables and age in the entire population, only the pulp width at points $\mathrm{B}$ and $\mathrm{C}$ had a significant inverse correlation with age. The pulp width/root width ratio also presented an inverse correlation with age. In other words, pulp width decreases or root width increases with age. Limdiwala et al. [11] evaluated maxillary and mandibular central and lateral incisors and second premolars on the panoramic radiographs of 20- to 55-year-olds; they found a significant

Table 2. Sensitivity, specificity, PPV, and NPV for the evaluated parameters

\begin{tabular}{|c|c|c|c|c|c|c|}
\hline Parameter & Cut Off Point (mm) & Sensitivity (\%) & Specificity (\%) & **PPV (\%) & $* * * N P V(\%)$ & Accuracy \\
\hline Tooth length & 27.5 & 74.4 & 64.7 & 61.7 & 76.7 & 68.9 \\
\hline Root length & 18.65 & 74.4 & 62.7 & 60.4 & 76.2 & 67.7 \\
\hline Pulp length & 22.15 & 74.4 & 62.7 & 60.4 & 76.2 & 67.7 \\
\hline Root width point $A$ & 6.55 & 70.2 & 72.5 & 70.2 & 86 & 77.7 \\
\hline Pulp width point $A$ & 1.35 & 69.2 & 72.5 & 65.9 & 75.5 & 71.1 \\
\hline Root width point B & 5.75 & 76.9 & 68.6 & 65.2 & 79.5 & 72.2 \\
\hline Pulp width point B & 1.15 & 60 & 64.7 & 60 & 73.3 & 66.6 \\
\hline Root width point $C$ & 4.85 & 74.4 & 76.5 & 70.7 & 79.6 & 75.5 \\
\hline Pulp width point $C$ & 0.85 & 78.4 & 74.5 & 68.6 & 72.7 & 71.1 \\
\hline
\end{tabular}


correlation between age and the root length of maxillary teeth. Other variables had an inverse correlation with age.

Paewinsky et al. [9] evaluated the panoramic radiographs of 6 teeth in 14- to 81-year-olds. They found no significant correlation between age and longitudinal ratios; however, the transverse ratio of pulp width/root width, at points $A$ and $B$, was significantly associated with age. Pulp width/root width, at point $\mathrm{A}$, in all teeth, except for mandibular premolars, was significantly correlated with age. Pulp width/root width, at point B, in mandibular premolars, had a significant correlation with age as well.

Similarly, Patil et al. [12] found a strong inverse association between age and pulp width/root width at points $\mathrm{B}$ and $\mathrm{C}$ on the PA radiographs of maxillary central incisors; this finding was consistent with ours. The relevant data indicate that these ratios may be used for age estimation. Bosmans et al. [13] found an inverse correlation between age and pulp length/root length, pulp length/tooth length, and pulp width/root width at points $\mathrm{A}, \mathrm{B}$, and $\mathrm{C}$. The standard error was equal to 8.1 years for age estimation based on the mandibular canine tooth, i.e., in line with our findings. Accordingly, it suggests that these ratios could be used for age estimation. Rajpal et al. [3] evaluated 6 maxillary and mandibular teeth. They found a significant correlation between all the measured ratios and age.

The standard error of estimation was found to be 6.4 years. However, the correlation between tooth length/ root length and age was not significant. Cameriere et al. [4] reported a significant correlation between the pulp volume/tooth volume of canine teeth and age in 29- to 79 -year-olds with a standard error of 5.4 years. Nitin Agarwal et al. [14] evaluated individuals aged $>20$ years and observed no significant relationship between tooth length, pulp length, root width, and pulp width at points $\mathrm{A}, \mathrm{B}$, and $\mathrm{C}$, and age; however, root length was significantly correlated with age.

The lack of a correlation between other measured factors and age in our study could be attributed to a small sample size, the applied methodology, the accuracy of conducted measurements, or the role of confounders, such as oral hygiene, dental care, and nutrition.

\section{Conclusion}

A significant correlation was noted between age and pulp width at points $\mathrm{B}$ and $\mathrm{C}$. Thus, these parameters could be used for age estimation in the adult population.

\section{Ethical Considerations}

\section{Compliance with ethical guidelines}

This study approved in ethical committee of Tehran University of Medical Sciences (Code: 93-11-22-3003).

\section{Funding}

This research was extracted from the MD thesis of the third author, Department of Forensic Medicine, School of Medicine, Tehran University of Medical Sciences.

\section{Authors' contributions}

All authors contributed in preparing this article.

\section{Conflict of interest}

The authors declared no conflicts of interest.

\section{References}

[1] Thali MJ, Brogdon BG, Viner MD. Brogdon's forensic radiology. CRC Press; 2010. [DOI:10.1201/b10323]

[2] Silver WE, Souviron RR. Dental autopsy. CRC Press; 2009. [DOI:10.1201/9781420070163]

[3] Rajpal PS, Krishnamurthy V, Pagare SS, Sachdev GD. Age estimation using intraoral periapical radiographs. Journal of Forensic Dental Sciences. 2016; 8(1):56-7. [DOI:10.4103/09751475.176955] [PMID] [PMCID]

[4] Cameriere R, Ferrante L, Belcastro MG, Bonfiglioli B, Rastelli $\mathrm{E}$, Cingolani M. Age estimation by pulp/tooth ratio in canines by peri-apical X-rays. Journal of Forensic Dental Sciences. 2007; 52(1):166-70. [DOI:10.1111/j.1556-4029.2006.00336.x] [PMID]

[5] Senn DR, Stimson PG. Forensic dentistry. CRC press; 2010. [DOI:10.4324/9780429292767]

[6] Taylor J, Kieser J. Forensic odontology: Principles and practice. Hoboken, New Jersey: John Wiley \& Sons; 2016. [DOI:10.1002/9781118864418]

[7] Cameriere R, Cunha E, Wasterlain SN, De Luca S, Sassarol E, Pagliara F, et al. Age estimation by pulp/tooth ratio in lateral and central incisors by peri-apical X-ray. Journal of Forensic and Legal Medicine. 2013; 20(5):530-6. [DOI:10.1016/j. jflm.2013.02.012] [PMID]

[8] Kapoor N, Kothari P, Shukla RK, Mishra SD, Badiye A. Age estimation from tooth-pulp area ratio: A preliminary study. La Revue de Médecine Légale. 2019; 11(1):11-4. [DOI:10.1016/j. medleg.2019.11.003]

[9] Paewinsky E, Pfeiffer H, Brinkmann B. Quantification of secondary dentine formation from orthopantomograms-: A contribution to forensic age estimation methods in adults. International Journal of Legal Medicine. 2005; 119(1):27-30 [DOI:10.1007/s00414-004-0492-x] [PMID] 
[10] Jeevan MB, Kale AD, Angadi PV, Hallikerimath S. Age estimation by pulp/tooth area ratio in canines: Cameriere's method assessed in an Indian sample using radiovisiography. Forensic Science International. 2011; 204(1-3):209-e1. [DOI:10.1016/j.forsciint.2010.08.017] [PMID]

[11] Limdiwala PG, Shah JS. Age estimation by using dental radiographs. Journal of Forensic Dental Sciences. 2013; 5(2):11822. [DOI:10.4103/0975-1475.119778] [PMID] [PMCID]

[12] Patil SK, Mohankumar KP, Donoghue M. Estimation of age by Kvaal's technique in sample Indian population to establish the need for local Indian-based formulae. Journal of Forensic Dental Sciences. 2014; 6(3):166.

[13] Bosmans N, Ann P, Aly M, Willems G. The application of Kvaal's dental age calculation technique on panoramic dental radiographs. Forensic Science International. 2005; 153(23):208-12. [DOI:10.1016/j.forsciint.2004.08.017] [PMID]

[14] Agarwal N, Ahuja P, Sinha A, Singh A. Age estimation using maxillary central incisors: A radiographic study. Journal of Forensic Dental Sciences. 2012; 4(2):97-100. [DOI:10.4103/0975-1475.109897] [PMID] [PMCID] 